\title{
MCNP6 unstructured mesh application to estimate the photoneutron distribution and induced activity inside a LinAc bunker
}

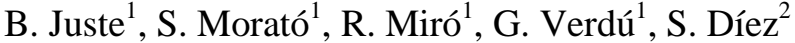 \\ (1) Instituto de Seguridad Industrial, Radiofísica y Medioambiental (ISIRYM). \\ Universitat Politècnica de València. Camí de Vera s/n. 46022. Valencia, Spain. \\ (2) Hospital Clínic Universitari de Valencia \\ Av. de Blasco Ibáñez, 17, 46010 València \\ *Corresponding author: Belén Juste \\ (Tel:+34963877635, Fax:+34963877639, e-mail: bejusvi@iqn.upv.es)
}

\begin{abstract}
Unwanted neutrons in radiation therapy treatments are typically generated by photonuclear reactions. High energy beams emitted by medical linear accelerators (LinAcs) interact with high atomic number materials situated in the accelerator head and release neutrons.

As photoneutrons scatter easily, they can leave the planned radiation field and be spread throughout the treatment bunker, contributing patients to receive a low (but non-negligible) dose, which is not estimated by treatment planning systems. Moreover, since neutrons have a high relative biological effectiveness, even low neutron doses may imply significant exposure of patients. It is also important to study activated radioisotopes for radiation protection purposes, as persons not present during irradiation (e.g. medical staff) may be exposed to them even when the accelerator is not operating.

The Monte Carlo code MCNP6 has been used in this work for quantifying the neutron contamination inside the treatment room. Walls and LinAc head activation processes by these neutrons have also been estimated by simulation. To that, a detailed model of particles transport inside the bunker has been carried out using a meshed geometry model. The LinAc studied is an Elekta Precise electron accelerator with a treatment photon energy of $15 \mathrm{MeV}$.
\end{abstract}

\section{INTRODUCTION}

High energy LinAcs used in external radiation therapy have several advantages including lower skin dose and higher dose rate at deep sighted tumors. High-energy photons generated by LinAcs with the energies higher than 8-10 MV interact with high atomic number materials, such as tungsten and lead located inside the accelerator head and can produce undesirable neutrons through photonuclear reactions. This is the case of the target, primary collimator, leaves and jaws of the Elekta Precise, which are made mostly of tungsten. The average energies of neutrons produced in this LinAc components are close to those of fission neutrons and have longer penetration depths than the primary photons and are therefore a major issue in terms of radiation protection.

As photoneutrons scatter easily, they can leave the planned radiation field and be spread throughout the treatment bunker, contributing patients to receive a low but non-negligible dose, which is not estimated by treatment planning systems. Moreover, according to ICRP Report No. 60, [1] the radiation weighting factor (WR) for neutron are quite large and it produce a quite significant dose equivalent in patients. Therefore, for optimizing the treatment conditions, it is important to take into account photoneutron contamination.

The photonuclear effect is based on the expulsion of a neutron (among other particles) from the nucleus, leading to, in most cases radioactive nuclei. The primary production of neutrons comes from $(\gamma, \mathrm{n})$ reaction, although it also exist a fewer contribution from $(\gamma, \mathrm{Pn})$ and $(\gamma, 2 \mathrm{n})$ reaction if the energy allows it.

The produced neutron from the $(\gamma, \mathrm{n})$ photonuclear process can induce additional transmutation of the surrounding material. This activated material can further decay through the $(n, \gamma)$ process, emitting additional gamma. 
These activation products may give rise to an increased irradiation of persons not present during irradiation (e.g. medical staff or technicians) which may be exposed to them even when the accelerator is not in operation. Elements in the air will also be activated but this is of minor importance.

The neutron dose distribution inside the treatment room is influenced by many factors such as the geometry of the room, and the neutron energy. According to this, a detailed model of particles transport inside the bunker has been carried out using Monte Carlo simulations. A second objective of this work is to include in the simulation a methodology for quantifying the activation of bunker walls and LinAc head.

Ambient dose calculations were calculated inside the bunker. Obtained results confirm that neutron dose received by patients is not negligible and should be taken into account. The photoneutrons produced can result in unwanted dose around $0.1-1 \%$ of the planned dose. Even if this extra dose may not be severe to patients, it could become serious exposure to daily workers, so future works will emphasize more on exposure to workers.

A second objective of this work is to include in the simulation a methodology for quantifying the activation of LinAc head components and bunker walls. This study has also used the MCNP6 code to show that some building materials become activated after irradiation. Using a detailed model of the medical accelerator, the photon and neutron activation in any location in the treatment room has been computed. The approach described in this paper is useful to prevent the overexposure of patients and medical staff.

One of the novelty of this work is the use of the latest version of MCNP, which has the ability to use unstructured meshes created with Abaqus/CAE as geometry descriptions. This improvement allows mechanical engineering models to be used in place of legacy constructive solid geometry descriptions, which are far less sophisticated and unable to reproduce complex geometries with the same accuracy level as meshes. Using Paraview for visualization the results calculated by MCNP6 is a major advantage over the previous ability to view results in two-dimensional space with MCNP's native plotter. This work proves that modelling via unstructured mesh allows to perform calculations faster and more precise.

Several authors have centered their studies in this field for different installations and machine types over the past 20 years [2], [3], [4] concluding that patients receive an expected additional dose around $0.5-5 \mathrm{mSv} / \mathrm{yr}$. There is no data for the Elekta Precise linear accelerator and its bunker, therefore this work studies the induced dose by a 15 MV photon beam inside the bunker including the adjacent control room around that LinAc head. The methodology described can be exported to any other LinAc machine and bunker geometry.

\section{METHODS AND METHODOLOGY}

In previous works, the LinAc photon beam production ( 6 and $15 \mathrm{MeV})$ was studied in detail until the Monte Carlo simulation dose distributions agreed with experimental measurements in a water tank [4]. In a second step, neutron production and activation inside LinAc head was also simulated. According to these studies, the most important component for photoneutron production is the target block with a contribution of about $25 \%$ of the total photoneutron production.

The problem is aggravated if high density materials are embedded in the walls, intended for shielding photons, but at the same time act as sources of photoneutrons as well.

In this work, we use those validated studies to investigate the neutron distribution inside an Elekta Precise LinAc treatment room, which is essential for radiation protection consideration. We also identify the induced activation products generated in the bunker walls and the LinAc itself.

\section{A. Monte Carlo Simulation}

The Monte Carlo method is widely used for simulating particle transport through different materials, and recording tallies (results) of the particles interactions. The code used in this work is Monte Carlo N-Particle (MCNP version 6) [5], which is a general-purpose Monte Carlo transport code developed at Los Alamos National Laboratory and includes extensive photonuclear reaction as well as neutron transport models additionally to electron-photon transport.

Direct measurements of neutron fluence and spectra in a medical accelerator room require complex measurement techniques and can often be time-consuming and rigorous. Monte Carlo methods, on the other hand, have become alternative methods since they are quicker and more flexible than taking measurements.

The use of meshed geometries, presents the advantage of a higher accuracy in the geometry modelling which is reflected in an increased absorbed dose estimation precision. Unstructured meshes enable the use of cells of different 
types and/or different size, allowing complex geometries and optimizing the number of cells used according to the needs. That is why, for the present work, we have used unstructured grids imported from ABAQUS/CAE. After MCNP6 simulation, the three-dimensional dose distribution can be shown using visualization software ParaView.

\section{1) Elekta model}

The detailed geometry of the radiotherapy treatment head unit Elekta Precise equipped with an 80-leaf MLC (operating with a $15 \mathrm{MeV}$ photon beam), has been accurately implemented in a Monte Carlo model according to the manufacturer data (Figure 1).

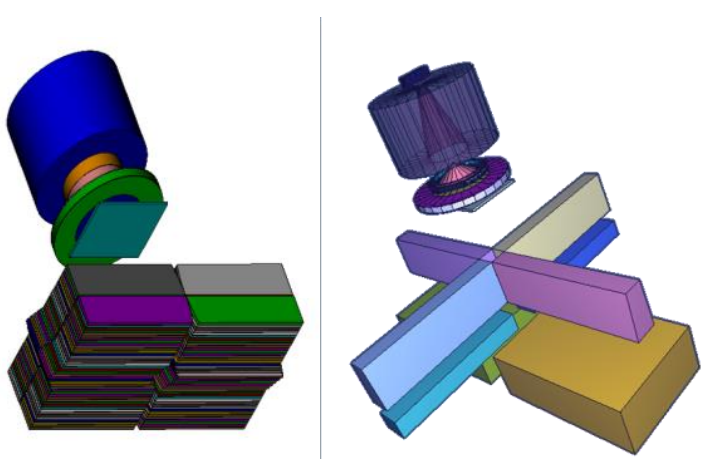

Figure 1. Elekta Precise geometry model.

The validation of the MLC LinAc Monte Carlo model was previously carried out using depth dose curves in a water phantom, measured at the hospital Clínic Universitari de Valencia [4].

In this work, we show results for 0 degrees gantry angle, using a squared field size formed by MLC of $20 \mathrm{~cm}$ x 20 $\mathrm{cm}$. We selected this broad field size to analyze a unfavorable case regarding scatter and doses. The source-to-surfacedistance (SSD) was $100 \mathrm{~cm}$. Figure 1 presents two sketches of the geometry of the Elekta model.

The bunker geometry was modeled according to technical drawings. As shown in figure 2, the door consists of three layers, one made of lead, a second one made of polyethylene and the other polyethylene borate ( $5 \%$ boron).

To attenuate the high-energy photons, the walls of the LinAc bunker are made of thick concrete, (density $\rho=2.43$ $\left.\mathrm{g} / \mathrm{cm}^{3}\right)$, with the exception of some extra shields, whose material are Ledite XN-288 $\left(\rho=4.8 \mathrm{~g} / \mathrm{cm}^{3}\right)$ and Ledite XN$240\left(\rho=3.8 \mathrm{~g} / \mathrm{cm}^{3}\right)$. Ledite is a concrete and steel mixture that combines excellent structural strength with a high level of resistance to radiation. The mass proportion of steel in Ledite XN-240 is 0.52 times the concrete mass. In Ledite $\mathrm{XN}-288$ the percentage is higher, 1.56. lead or steel compored to the case where pure concrete barriers are used. The

Figure 2 shows the bunker treatment geometry model and its generated mesh.
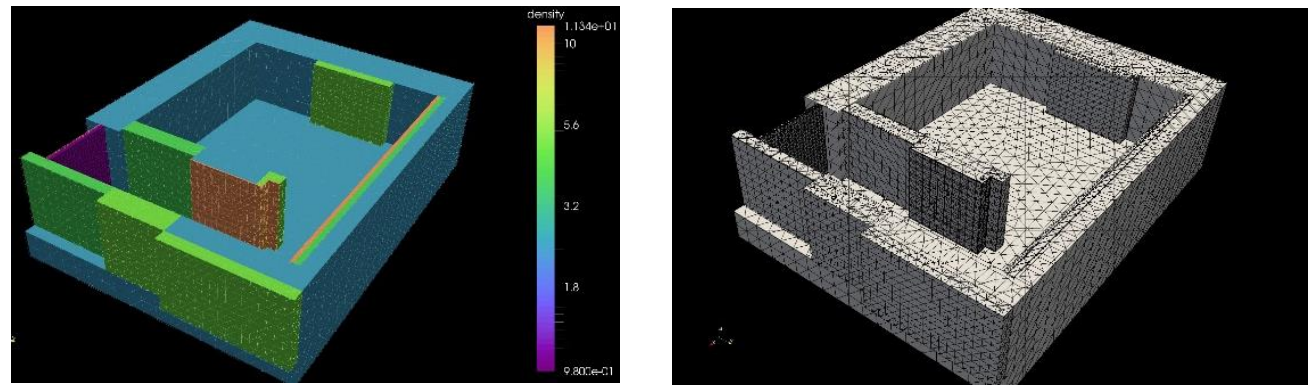

Figure 2. Model and mesh of the Elekta Precise treatment room.

The results grid size was of about $2 \mathrm{~cm}$ in each direction. For mesh grid calculations FMESH4 tally was used including the DE/DF card (flux to dose conversion factors). 
To study the activation processes, the volume flux tally used determines the track length of a particle integrated over time, volume, and energy. The output is then divided by the total volume of the cell, resulting in units of particles $/ \mathrm{cm}^{2}$. The estimation of the track length is reliable since there are often many tracks in a cell resulting in many contributions to the tally. The volume flux tally can be modified by using a multiplier card (FM card) to obtain activated atom volume concentrations. The FM card allows several different multipliers to be used with the neutrons $/ \mathrm{cm}^{2}$ output. First an atom concentration in the volume is determined.

In order to speed up the calculations, the simulation of the particles inside the bunker has begun in a phase space generated previously at the exit of the jaws.

$10^{9}$ primary particles were simulated in order to obtain an uncertainty in all evaluated points below $3 \%$.

\section{RESULTS}

\section{1) Photoneutron distribution}

Figure 3 shows the relative fluence distribution (top view) inside the treatment bunker for a $142 \mathrm{~cm}$ height plane predicted by the Monte Carlo simulation. Figure 3a shows the photon and electron contribution to fluence distribution inside the room. On the other hand, figure $3 \mathrm{~b}$ takes into account just the contribution of photoneutrons to the fluence. Results are represented in a relative logarithmic scale that reaches from $10 \mathrm{e}^{-8}$ to $10 \mathrm{e}^{-12}$ part $/\left(\mathrm{cm}^{2} \cdot \mathrm{seg}\right)$.

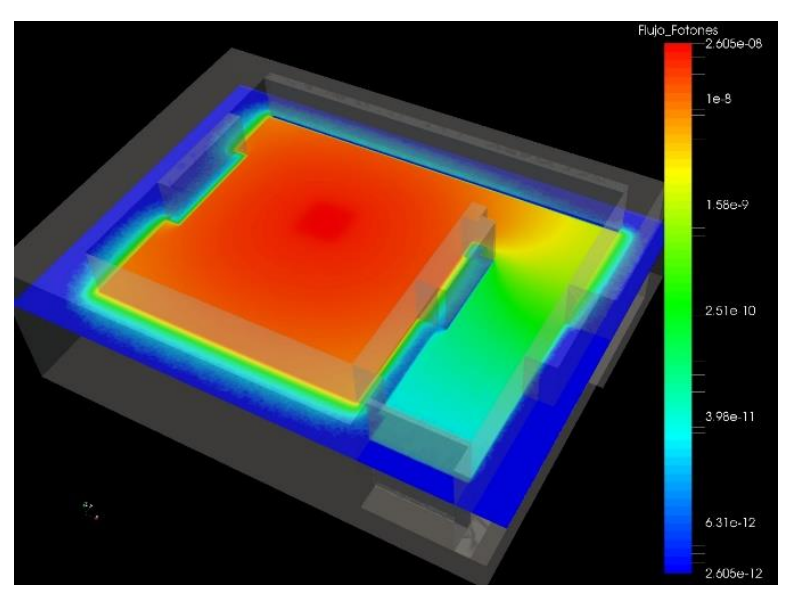

(a)

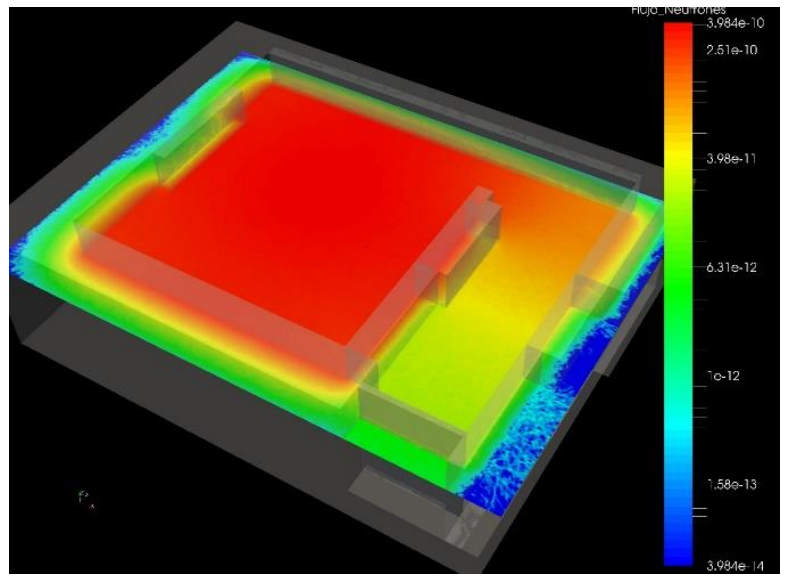

(b)

Figure 3. (a) Top view of fluence distribution due to photons for a $142 \mathrm{~cm}$ height plane.

(b) Top view of fluence distribution due to photoneutrons for a $142 \mathrm{~cm}$ height plane.

Figure 4a shows the photon contribution to absorbed dose distribution inside the room. On the other hand, figure $4 \mathrm{~b}$ takes into account just the contribution of photoneutrons to the dose. Results are expressed in a relative logarithmic scale that reaches from $10 \mathrm{e}-11$ to $10 \mathrm{e}-20 \mathrm{MeV} /(\mathrm{gr} \cdot \mathrm{seg})$. 


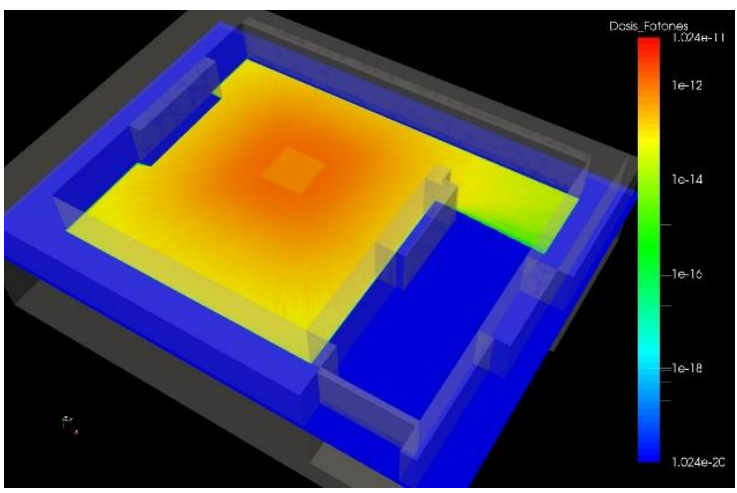

(a)

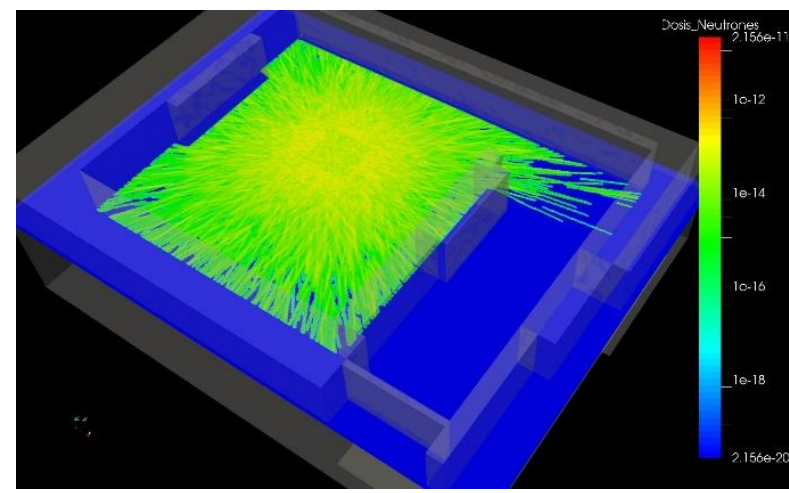

(b)

Figure 4. (a) Top view of equivalent dose distribution due to photons for a $142 \mathrm{~cm}$ height plane.

(b) Top view of equivalent dose distribution due to photoneutrons for a $142 \mathrm{~cm}$ height plane.

The produced photoneutrons result in unwanted dose (around 1\% of the planned dose) to not only the patient inside the LinAc treatment room but also to oncology staff and the public outside the treatment room [6], [7].

It should also be taken into account that these values do not include effective dose due delayed photons due to activation in the concrete.

\section{2) Activation products analysis}

The primary and secondary barriers in the treatment bunker are several tons of concrete that are activated during a patient treatment and lifetime of the facility, behaving as a $4 \pi$ source that deliver a dose in the bunker lasting up to few years after the treatment.

To study the activation processes we will show results for a $0^{\circ}$ gantry rotation. Figure 5 presents the resultant gamma-rays energies from the simulation activation results with the MCNP6 code. It presents the comparison of results using the activation FM card associate to F4 volumetric tally for each reaction. Results are presented in relative count rate.

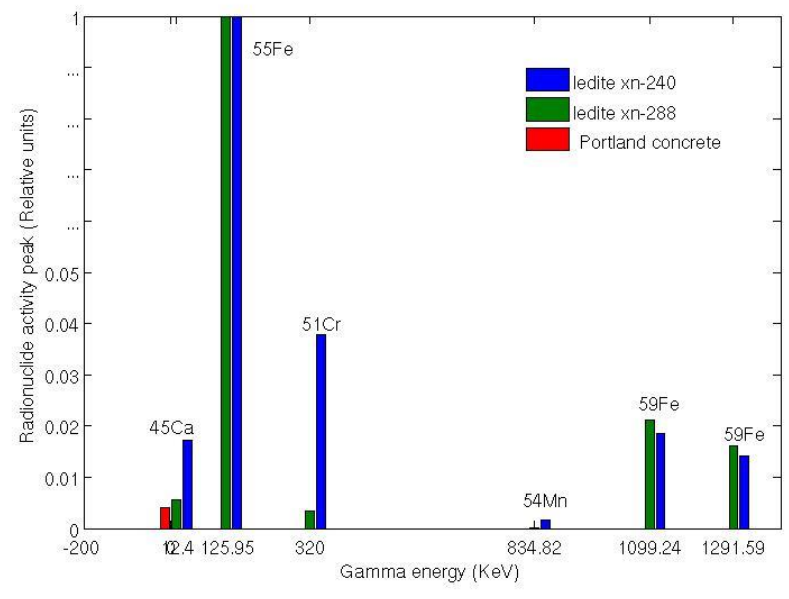

Figure 5. Induced activation products at the bunker walls

It can be seen that the reaction $54 \mathrm{Fe}(\mathrm{n}, \gamma) 55 \mathrm{Fe}$ is the one which generates the highest radionuclide activity peak This event comes from the steel component of Ledite material In comparison, other important activated product for Ledite $\mathrm{XN}-240$ material is $51 \mathrm{Cr}$, which comes from $50 \mathrm{Cr}(\mathrm{n}, \gamma) 51 \mathrm{Cr}$ reaction, although it is two orders of magnitude lower than $55 \mathrm{Fe}$. 
In figure 5 is shown the pulse height peak of the radioisotopes induced in the walls, where the main peaks are $125.95 \mathrm{KeV}$ emitted by $55 \mathrm{Fe}$ (2.7 years half-life), $820 \mathrm{KeV}$ emitted by $51 \mathrm{Cr}$ and 1099.24 and $1291.59 \mathrm{KeV}$ that are emitted by $59 \mathrm{Fe}[8]$.

Figure 6 presents the simulation results inside the LinAc head. Results are presented in relative count rate. It can be seen that the reaction $186 \mathrm{~W}(\mathrm{n}, \gamma) 187 \mathrm{~W}$ is the one which generates the highest radionuclide activity peak. Tungsten is the main component of target, primary collimator, jaws and leaves. In comparison, other important activated product is $56 \mathrm{Mn}$, although it is two orders of magnitude lower tan $187 \mathrm{~W}$. This last isotope is generated mainly in the flattening filter.

Activity products in air (41Ar) has also taken in to account in the simulation, but results obtained demonstrated that its consideration can be negligible.

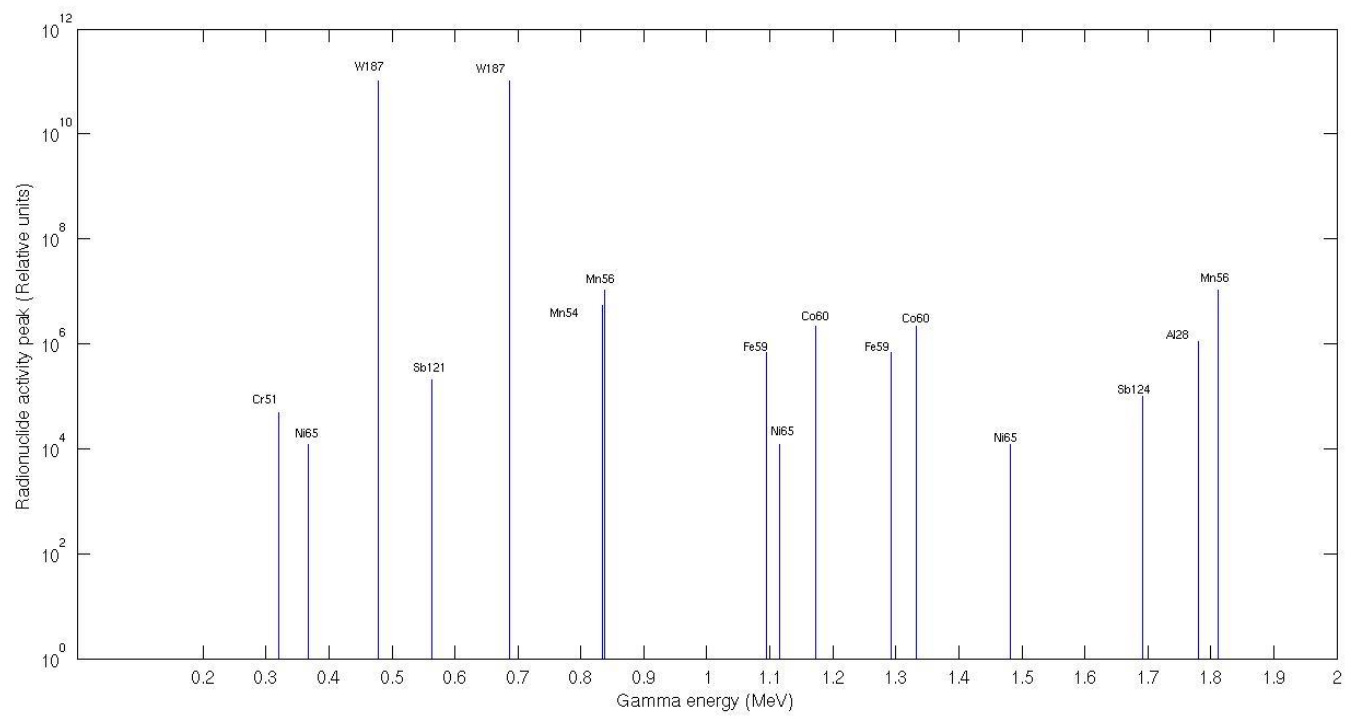

Figure 6. Activated products generated in the linac head and calculated by Monte Carlo simulation.

\section{CONCLUSION}

A computer model of an Elekta LinAc head and a bunker has been successfully completed. Using MCNP6, the photon, electron and neutron phase space at the end of the LinAc head was calculated. This phase space was then used as a radiation source in the modelled bunker. Ambient dose calculations were calculated inside the bunker showing that neutron dose received by patients is not negligible and should be taken into account. The photoneutrons produced can result in unwanted dose (around $0.1-1 \%$ of the planned dose). Even if this extra dose may not be severe to patients, it could become serious exposure to daily workers, so future works will emphasize more on exposure to workers.

This study has also used the MCNP6 code to show that some building materials become activated after irradiation. Using detailed model of medical accelerators, the photon and neutron activation in any location in the treatment room has been calculated.

Neutron calculations will be verified with experimental measurements using Bonner spheres with a 6LiI detector in the next future.

\section{ACKNOWLEDGMENT}

We would like to thank the "Hospital Clínic Universitari de Valencia" for the given help. This work has received support via "Programa para la innovación e incentivación" from Universitat Politècnica de València "INNOVA 2012”. 


\section{REFERENCES}

[1] ICRP, 1991. 1990 Recommendations of the International Commission on Radiological Protection. ICRP Publication 60. Ann. ICRP 21 (13).

[2] N. L. Powell, A. Newing, M. A. Bullen, C. Sims and F. S. Leaton, "A radiation safety survey on a CLinAc-20 linear accelerator", Phys. Med. Biol. Vol. 32, pp.707-18, 1987.

[3] J. R. Rawlinson, M. K. Islam and D. M. Galbraith, "Dose to radiation therapists from activation at high-energy accelerators used for conventional and in tensity-modulated radiation therapy", Med. Phys vol. 29, pp.598-608, 2002.

[4] L. Ahlgren and L. E. Olsson, "Induced activity in a high-energy accelerator", Phys. Med. Biol. Vol. 33, pp. 351-354, 1988.

[5] B. Juste, R. Miró, et al., "Experimental validation of an accurate multileaf collimation (MLC) LinAc simulated by Monte Carlo", Applied Radiation and Isotopes vol. 68, pp. 432-436, 2010.

[6] Monte Carlo team, "MCNP - A General Monte Carlo NParticle Transport Code, Version 6", Los Alamos National Laboratory.

[7] A. Zanini, E. Durisi, F. Fasolo, et al. « Monte Carlo simulation of the photoneutron field in LinAc radiotherapy treatments with different collimation systems”, Phys Med Biol, vol. 49, no. 4, pp. 571-582, 2004.

[8] A. Almen, L. Ahlgren and S. Mattsson, "Absorbed dose to technicians due to induced activity in linear accelerators of radiation therapy", Phys. Med. Biol. Vol. 36, pp. 815-22, 1991.

[9] C. M. Lederer and V. S. Shirley, "Tables of Isotopes” New York: Wiley, pp 1-152, 1978. 